\title{
Materials of the round table "Phase transitions and critical phenomena: past, present, and future"
}

\begin{abstract}
The paper contains materials of the discussion concerning phase transitions and critical phenomena which took place during the Workshop on modern problems of soft matter theory (August 27-31, 2000, Lviv, Ukraine).
\end{abstract}

Key words: phase transitions, critical phenomena

PACS: 01.30.Rr, 05.70.Fh, 64.

\section{Is that true that all principal work in the phase transitions the- ory has already been done up to the middle 80 -ies and there is neither new physics there to be explained nor a need to apply efforts to theoretical studies?}

Yurij Holovatch. Dear colleagues, I have the pleasure to open our round table during which we will have a possibility to discuss past, present and possible future of the field most of us are currently interested in: the phase transitions theory. Perhaps it is hard to name another branch of modern physics which was so essentially changed during last 30 years as the theory of phase transitions and critical phenomena. One can say even more: having appeared in a form of different variants of a mean field theory for describing critical points in liquids and equilibrium phase transitions in problems of statistical physics and thermodynamics, the theory of phase transitions and critical phenomena now appears to be an interdisciplinary science as, say, the theory of vibrations. Also, the sphere of notions contained in the term "critical phenomena" has been essentially broadened. Aside from traditional critical points in liquids and equilibrium thermodynamic 2nd order phase transitions, this term includes non-equilibrium dissipative phase transitions, percolation phenomena as an example of a geometrical phase transition when there appears an infinite (percolating) cluster in a system. Properties of long flexible polymer chains in good solvents are described in terms of critical phenomena as well. One can go on mentioning different examples of phase transitions and critical phenomena but let me rather attract your attention to the following question: what is your opinion regarding the state of the art in this matter and especially regarding possible future 
developments? To start, I just want to mention two polar opinions existing in the field: one of them states that it is a fascinating part of physics still actively developed and there is a lot of work to be done there both on the fundamental level as well as in applications. The other opinion is that all principal work in the phase transitions theory has already been done by the middle of the 80-ies and there is neither new physics there to be explained nor need to apply efforts in theoretical studies. You are welcome to give your support or objections to any of these opinions as well as to tell us what in your mind is happening and will be happening in the field of phase transitions and critical phenomena.

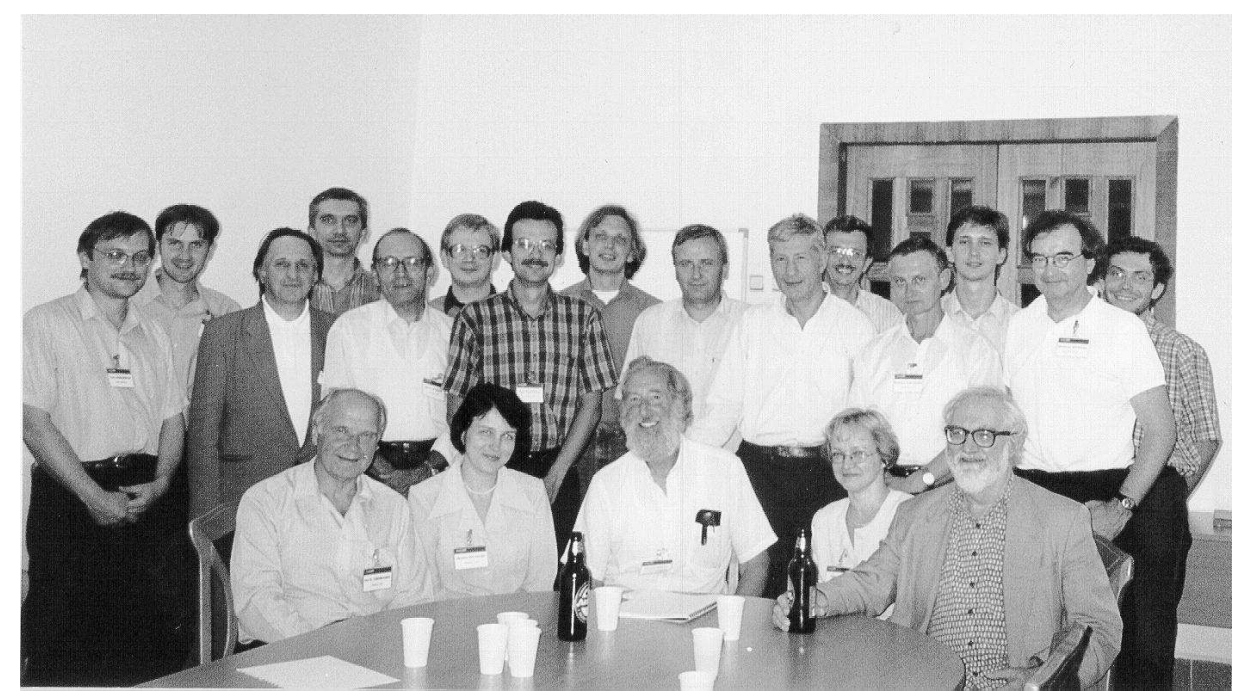

Participants of the round table "Phase transitions and critical phenomena: past, present, and future" (Lviv, August 30, 2000). Seated (from left to right): I.Yukhnovskii, O.Patsahan, M.Schick, A.Ciach, G.Stell. Standing: Yu.Dublenych, M.Dudka, R.Levitskii, O.Ivankiv, I.Stasyuk, O.Velychko, Yu.Holovatch, C. von Ferber, A.Zagorodnii, O.Bakai, I.Pylyuk, M.Kozlovskii, R.Sokolovskii, W.Schröer, T.Yavors'kii.

\section{The future of critical phenomena is its glorious past!}

Michael Schick. Well, I will start out by playing devil's advocate. Of the two positions you have outlined, I would take the second. As you say, the theory of phase transitions was basically completed by the middle of the eighties. It was a wonderful time. But the phenomena are now well understood, and I would not counsel students to go into it. Basically one is now, in my view, cleaning up details, and that is not the kind of thing which students should be doing. They should be going into new areas. Critical phenomena was a fantastically exciting field TWENTY years ago. In terms of the future, I think it will be like many other areas of condensed matter physics which have been studied; it's been understood, so move on! I don't think that one should try to keep students in critical phenomena just because we had such a good time and learned so much from it. It was fantastic, it was great! But it is 
basically well understood. As I said, if I were to counsel students, I would say that you should learn this material because it's beautiful. It's a wonderful subject! But I don't think that's where you should spend your time in research. You should go into something which is not so well understood, and which presents new problems, hopefully of the same order that critical phenomena presented twenty years ago. I think it would be a real mistake to try and continue in this area. Obviously I may be wrong. But to summarize, I would say that the future of critical phenomena is its glorious past.

\section{You want to find out very basic principles but you find out how to apply theory to real systems!}

Reinhard Folk. I would like to take the other point of view. Of course the theory of critical phenomena is understood in principal, but I think theory has proceeded to a stage where you perform quantitative calculations. One wants to test theory within high precision. You might say that this is not so interesting, but on the other hand if you use such a complicated theory, you have to be sure that it describes the phenomena precisely. Take as an example the superfluid phase transition. There you measure in space the values of the critical exponents and then compare them with theory. This is similar to the tests of the theory in elementary particles physics. That is one point. The other point is, that one uses the RG theory and equivalent theories for applications in real systems. There are many problems which are solved only by simple approaches, e.g. first order approximations and which are not sufficient. As examples I like to mention crossover effects in complex fluids, where you have to consider the influence of two different types of critical behaviour or magnetic systems with impurities. Or one may study liquids measuring transport coefficients in nonequilibrium situations with finite heat currents or under shear. I also think that there are a lot of problems not solved below $T_{\mathrm{c}}$ because they are much more complicated than the equivalent problems above $T_{\mathrm{c}}$. So, in my opinion there is enough space for more work to do, also it's not of that basic kind that you say. You want to find out very basic principles but it is also of value to find out how to apply theory to real systems. So that's another type of problems in physics but it has its own value.

\section{The thing is to successfully marry the second virial coefficient and scaling theory.}

George Stell. Well, I think I agree with what Michael Schick says about critical phenomena, largely, but there was an implicit assumption in the way you started out, Michael. I mean you implicitly identify phase transitions and criticality. When I got to the scene in the early 1960's there were two separate things that were interesting. First there was the beginning of what became scaling theory, and homogeneity, and then renormalization-group theory. Second, there was an independent but equally 
interesting and important set of developments in liquid-state theory to predict with quantitative accuracy the phase transitions associated with going from gas to a liquid and to a solid. Those are associated with what happens in regions in the phase diagram that can be very far away from a critical point.

In other words, the theory of phase transitions is much broader than the theory of criticality, I would say. And that gave rise to a problem that proved to be much more difficult than people initially expected it to be, I think - the problem of marrying the results of thermodynamic perturbation theory that had been fine tuned by the early 1970 's to give very good global thermodynamics away from the critical point to the dictates of critical-point theory, which at about the same time had been developed to the point at which it could give very accurate thermodynamics in a very narrow region around the critical point. The initial engineering-oriented attempts to glue those two things together were all unsuccessful.

Roughly speaking, the reason they were unsuccessful goes like this, technically. Critical theory tells you that you have homogeneity or scaling of thermodynamics on all length scales. And homogeneity means you have something that will be true arbitrarily far away from a critical point, if you take it literally rather than as asymptotic condition, because homogeneity is intrinsically a global rather than a local concept. So the difficult thing was shutting off the homogeneity as one goes away from the critical region. And one HAS to do this in a successful theory - after all, the second virial coefficient never heard of homogeneity. The embarrassing thing was that it turned out to be very difficult to successfully marry the second virial coefficient and scaling theory, so to speak.

That's something that's been of continuing interest to me and to several other groups for many years, and only in the last decade or so it has been successfully coped with by us. For example by Reatto's Milano group and by my group, in theories that yield both structure factors as well as thermodynamics, as by other groups who deal only with the thermodynamics. And it's still a work in progress, because one wants to deal not only with argon and the Ising model but with the criticality of ionic systems and polar fluids and polymers and colloids and microemulsions etc., etc. Even when one is looking at what turns out to be an Ising-like critical point in such complex and soft-matter systems, the special symmetries of these systems give rise to new experimental twists to what one observes - different sorts of cross-over phenomena and the like. And in some of them, one has not only liquid-gas Ising-like criticality in disguise but the possibility of many other different types of critical or singular points - tricritical points, Lifshitz points, etc., etc. that one also wants to be able to treat in the context of globally accurate theories.

Over the last thirty years there has been remarkable progress in dealing with dense fluids of complex molecules away from critical points. Thirty years ago we knew already how to deal with simple fluids by introducing hard-sphere reference potentials and then adding soft tails to them as a perturbation, treating the hardsphere fluid by using things like the Percus-Yevick theory and the Carnahan-Starling approximation. Now, with about the same amount of accuracy and almost the same degree of analytic simplicity, we can treat dense fluids of arbitrarily long hard-sphere 
chains, or dendrimers, or star polymers, as long as they are composed of hard-sphere monomers. And we know how to do a perturbation theory for adding attractive tails to the monomer-monomer hard-sphere interaction. We now also have wonderfully simple and remarkably accurate theories for the thermodynamics of dense fluids of associating molecules, based on work by Wertheim and others that enable us to consider the association of hard-sphere particles into hard molecules, including the hard-chain polymers mentioned above. In fact, the development of such theories of association is a specialty of a number of the people who are here in Lviv.

One thing that has not been developed to a high level of accuracy is the thermodynamics and structure for reference potentials that are repulsive inverse-power potentials proportional to relatively low inverse powers (4 through 9 , say) of the distance between particle centers. Such potentials are appropriate monomer-monomer reference-system potentials for a number of soft-matter systems, and the excess thermodynamics of a soft-sphere system depends upon temperature and density only through a single variable. There was some nice work done on such models in the 1970's and early 80's but its use seems to have languished over the last decade compared to the use of hard-core reference systems.

Another thing that remains rather poorly developed is analytic theory in two dimensions. For example, there is no theory of hard disks as beautifully simple as the Percus-Yevick theory of hard spheres. This is because of deep technical reasons: the solutions of Weiner-Hopf type equations (or, for that matter, of the Helmholtz equation) have a more singular analytic structure (involving logarithms) in even dimensions than in odd dimensions. But I think more can be done on this problem than has been done, and it would be worth the effort.

Similar statements can be made about binary mixtures of hard spheres with nonadditive diameters in three dimensions. There have been some heroic assaults on this problem from time to time, but more are needed.

\section{The next century will be the century, when the interest of sci- entists will be shifted towards living systems.}

Alexander Chalyi. I would like to use a few minutes to speak about future applications of the theory of phase transitions to living systems, namely to the synaptic transmission or cell-to-cell communication. This phenomenon plays the same role in living systems as the intermolecular interactions in non-living systems. Actually the first paper "Phase Transitions in Finite-Size Systems and Synaptic Transmission" on this topic was reported by me and Dr. L.M.Chernenko at the Les Houches Workshop "Dynamic Phenomena at Interfaces, Surfaces and Membranes".

The convenient theory of cell-to-cell communication is commonly based on the ideas concerning chemical intermediaries (transmitter agents) securing transmission between two neurons in the synaptic cleft or between the motor neuron and muscle fibre in the neuro-muscular junction. The sequence of major events in cholinergic synapse are as follows: Acetylcholine $(A c h)$ - let us consider this typical transmitter - is synthesized and stored in spheroid vesicles, then releases and reacts with spe- 
cific acetylcholine receptors $\left(R^{*}\right)$ in active states. The formation of the transmitterreceptor complex $\left(A c h-R^{*}\right)$ produces conformal changes in the postsynaptic membrane and therefore the change in a membrane potential. Thus, it is the main process of transmitting the information from cell to cell, say, in a human brain. It is possible to write a scheme of biochemical reactions and corresponding kinetic equations describing the temporal evolution of concentrations of receptors $R^{*}$ and transmitterreceptor complexes $A c h-R^{*}$. It must be stressed that the process of Ach release is cooperative: about 107 Ach molecules are released simultaneously under the influence of one nerve impulse. Furthermore, such synchronous activation of such a large zone of receptors by Ach can be considered in detail as the process which is isomorphic to the critical phenomena in binary liquid mixtures near the critical mixing point.

So, it's a very important application of the phase transitions theory. We mostly speak about technical applications like displays on liquid crystals, etc. Without any doubt, these technical applications are very useful. Nevertheless, it seems to me that the next century will be the time when the interests of scientists (first of all physicists) will be shifted towards living systems.

And the last remark: the Chairman of the Les Houches Workshop, I mentioned above, was a famous French physicist P.G. de Gennes. It was exactly in February, 1991 and he received his Noble Prize several months later. In the foreword to the proceedings of this workshop ( DDnamic Phenomena at Interfaces, Surfaces and Membranes". Eds. D.Beysens, N.Boccara and G.Forgacs, New York, Nova Science Publishers, 1993) P.G.de Gennes started with the words "Biophysics is a Tower of Babel" and concluded: "I left Les Houches with a more optimistic view of biophysics. I am in fact convinced that nearly all physicists and chemists should have an education in biology - not only in the molecular aspects, which are usually invoked, but also a panorama of the living world - telling us repeatedly that Nature is much more inventive than us".

\section{The future for the phase transitions theory is the mixed theory: my mind + computer.}

Ihor Yukhnovskii. I would like to tell you about my view on these different works which were presented at our conference. You see, when we want to talk about the phase transition, we, of course, may use the mean field approximation and obtain this very nice coexistence curve (see figure 1) within the Van der Waals-like theory. We can also obtain the coexistence curve from numerical simulations but near the critical point this result might be wrong. It may be good far from the critical point, but near the critical point the fluctuations will grow and it will be difficult to reach it. The critical exponents in the critical point are non-trivial. What's to be done? Is it enough for us to use the mean field or, knowing that in this point the mean field is not valid, we have to build a new theory and to consider different complicated tasks concerning the phase transition on the base of this theory? As an example, I would like to very briefly sketch a very old problem, namely, the liquid-gas critical 


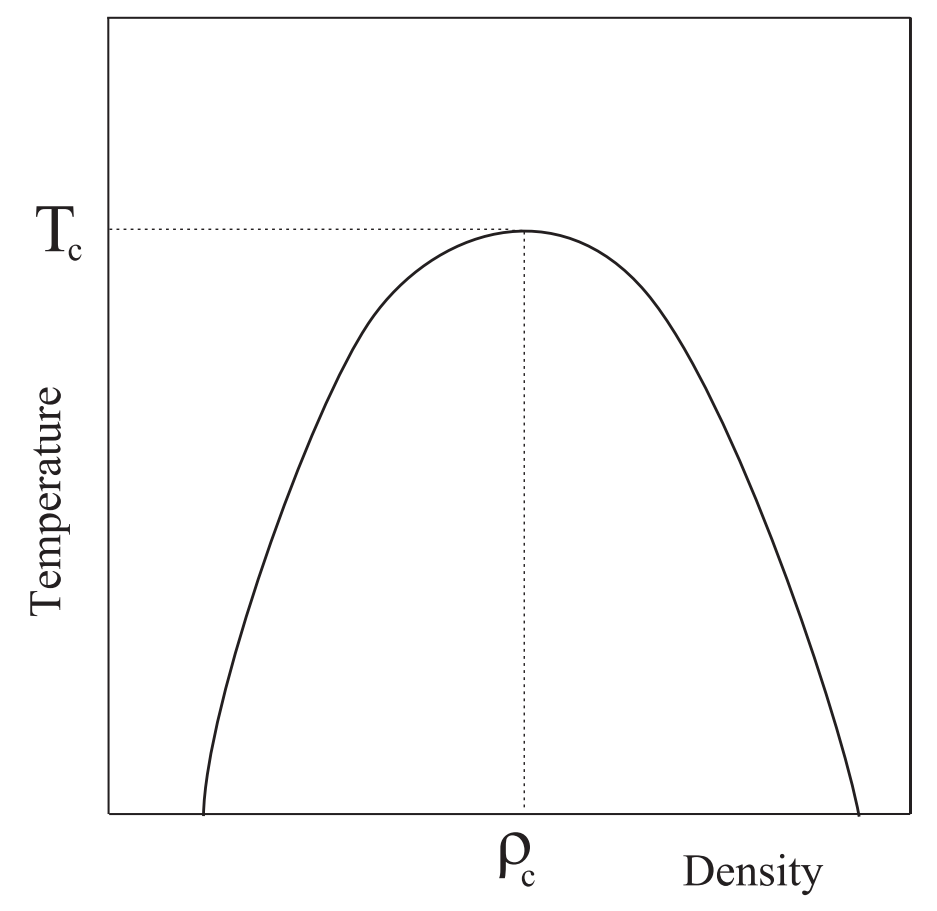

Figure 1. A coexistence curve and a critical point for a liquid-gas phase transition.

point. It was the most difficult task in the phase transition theory.

We have the Hamiltonian with the interaction:

$$
U\left(r_{i j}\right)=\psi\left(r_{i j}\right)+\phi\left(r_{i j}\right)
$$

consisting of two parts: $\psi\left(r_{i j}\right)$ describes repulsive forces, and $\phi\left(r_{i j}\right)$ is the attractive part which, for example, can be chosen in the form of the Lennard-Jones potential. Why does the phase transition occur? The phase transition is present because of a very long tail of the attraction in the Lennard-Jones potential. How shall we describe the phase transition? Of course, we can construct the free energy containing some additional variable. This variable is an order parameter and we can work with this free energy. Having the expression for the free energy we may talk about the phase transition. But this is a constructive theory, this theory is not rigorous. The phase transition can be described rigorously only if we use a proper phase space. We will call it the collective variables phase space. Among the variables in this phase space, there must be a variable which in some limit gives the macroscopic order parameter. For every task, for every physical problem there exists its own set of appropriate collective variables and we have to find such variables. To give an example, let us discuss the liquid-gas critical point. You know that in this case the order parameter is related to density. Therefore, we have to introduce some variables which are the modes of the density fluctuations:

$$
\hat{\rho}_{\vec{k}}=\sum_{i=1}^{N} \exp \left(-\mathrm{i} \vec{k} \vec{r}_{i}\right) .
$$


And the partition function may be written as:

$$
\Xi=\Xi_{0} \int(\mathrm{d} \rho) \exp \left[\beta \mu_{1} \rho_{0}-\frac{\beta}{2 V} \sum_{\vec{k}} \tilde{\phi}(k) \rho_{\vec{k}} \rho_{-\vec{k}}\right] J(\rho),
$$

where $\Xi_{0}$ is the hard sphere contribution: it is the grand partition function of the hard sphere reference system. The potential energy of attraction has the square form in the new variables and $J(\rho)$ is the most difficult part, the Jacobian of the transition from Cartesian coordinates to these new collective coordinates. Note that among these coordinates there is one which is connected with the order parameter, namely, $\hat{\rho}_{\vec{k}}$ for $k=0$. We get the following representation for $J(\rho)$ :

$$
\begin{aligned}
J(\rho)= & \int(\mathrm{d} \omega) \exp \left[\mathrm{i} 2 \pi \sum_{\vec{k}} \omega_{\vec{k}} \rho_{\vec{k}}\right] \\
& \times \exp \left[\sum_{n \geqslant 1} \frac{(-\mathrm{i} 2 \pi)^{n}}{n !} \sum_{\vec{k}_{1} \ldots \vec{k}_{n}} M_{n}\left(\vec{k}_{1}, \ldots, \vec{k}_{n}\right) \omega_{\vec{k}_{1}} \ldots \omega_{\vec{k}_{n}}\right],
\end{aligned}
$$

where $M_{n}\left(\vec{k}_{1}, \ldots, \vec{k}_{n}\right)$ is the density cumulant of $n$th order defined for the reference system. When you put all cumulants with $n \geqslant 2$ equal to zero you obtain the analogy of a mean field theory. This means that the critical exponents will be wrong, they will be the critical exponents of the mean field theory. If you want to obtain right critical exponents in all the region you should take into account $\mathcal{M}_{n}$ with $n=1,2,3,4$. And you will obtain the right theory, the right exponents, and you can obtain the correct thermodynamics.

The order parameter is defined by a collective variable $\rho_{\vec{k}}$ with $k=0$. The following theorem for the cumulants $M_{n}\left(\vec{k}_{1}, \ldots, \vec{k}_{n}\right)$ (4) can be proved: when one of the arguments $k$ is equal to zero, the cumulant is automatically equal to zero if the finite size system is considered. Only when the thermodynamic limit $N, V \rightarrow \infty$ is taken the cumulants $\mathcal{M}_{n}$ for $k=0$ are non-zero and can be expressed by some thermodynamic derivatives. This means that you should be careful when you study a finite system.

And finally, I want to say that new possibilities in the studies of phase transitions are expected from some "mixed" theory: my mind + computer, not only a computer without mind, not only mathematics without computer because the things are very complicated. And now about different problems, bioorganic problems and so on. You have seen during our conference that very complicated systems can be described by non-central forces. In the collective variables method we can consider any potential: symmetric, non-symmetric and so on. The theory will be more complicated but you have a computer, so you can work with such systems. To conclude, I believe that the most important thing in the theory of phase transitions is to have the method which is quite general and which allows one to obtain the correct values for critical exponents and makes it possible to obtain entire thermodynamic functions. 
Wolffram Schröer. I don't really want to continue the discussion about future of the critical phenomena. The situation we are facing in respect to the critical phenomena is to some extent reminiscent to the situation in quantum mechanics in the late twenties of last century when the basic equations were developed, however the tedious work to required understanding of chemistry which is still progressing was not done. I would rather ask just after you talk, Professor Yukhnovskii. How much is really done in your method? Have you really got the non-classical exponents in this method?

Ihor Yukhnovskii. Yes, of course, quite naturally, without any adjusting parameters, only knowing the interaction potential between particles you obtain all thermodynamical functions, critical exponents. They are of quantitative accuracy.

Michael Schick. The critical exponents in most phase transitions are known by now with high enough accuracy.

Ihor Yukhnovskii. Yes, we obtain the same critical exponents, of course.

Reinhard Folk. May I make some comments. The problem just mentioned by Professor Yukhnovskii is neither to calculate the value of the asymptotic critical exponents, nor the Van der Waals theory but it is the crossover between these two limiting behaviours. You have to find a method which is systematic and which can give both regions correct. And there are theories e.g. by Anisimov and Sengers who calculate this crossover in a phenomenological way. They can adjust the equation of state in such a way that they have correct asymptotic exponents together with correct amplitude ratios and that crosses over in the background to the classical equation of state. This could be the Van der Waals equation or any other equation. I think, it would be very important to prove, that with the method described by Professor Yukhnovskii you really can do the same. Then you have a theory which describes the crossover in a systematic way. This would be an improvement over all theories existing so far.

Alexander Chalyi. I would like to say that I know the very important results obtained in this method of collective variables. Rigorous transition from the microscopic Hamiltonian, or microscopic variables to order parameter variables, to collective variables through the Jacobian of this transition. This point is absent in any other approach. Because the starting point of the renormalization group theory is to use the effective Hamiltonian. And here the coefficients are obtained explicitly. This is the first.

The second. Concerning this extended equation of state, it seems to me, there are different wrong opinions that it must be the crossover between the so-called scaling and classical behaviour inside the critical region for reduced temperature variable $\left(T-T_{\mathrm{c}}\right) / T_{\mathrm{c}}$ between zero and one. But the existence of such a transition depends on the value of Ginzburg number. If the Ginzburg number is of order of one and more there is no region where the crossover occurs. If the Ginzburg number is smaller than one, then you must have the crossover.

Yurij Holovatch. I want to comment that in fact both of you are right. Because experimentally unobservable crossover region simply means that it is very narrow and of course its width depends on the microscopic features of a real system. The 
crossover is always present, but its width is system-dependent.

Reinhard Folk. There is already experimental evidence, comparing the critical behaviour of $\mathrm{Xe}$ and $\mathrm{He}^{3}$, that one finds within the so-called critical region deviations from the renormalization group results. These are caused by the effects which are background effects. So other lengths come into play already in this region and a variety of crossover phenomena can be observed.

Yurij Holovatch. So, the crossover scenario is system dependent. Please, Doctor Kozlovskii.

Mykhailo Kozlovskii. I would like to propose to your attention some remarks concerning the behaviour of non-universal quantities at the second order phase transition. In the collective variables method it is possible to calculate not only universal properties but non-universal ones. As you know the partition function can be represented in the following form:

$$
\begin{aligned}
Z= & C \mathrm{e}^{a_{0} N} \int \exp \left(-\frac{1}{2} \sum_{\mathbf{k} \in \mathcal{B}} d(k) \rho_{\mathbf{k}} \rho_{-\mathbf{k}}\right. \\
& \left.-\sum_{n \geqslant 2} \frac{a_{2 n}}{(2 n) !}(N)^{1-n} \sum_{\substack{\mathbf{k}_{1} \ldots \mathbf{k}_{2 n} \\
\mathbf{k}_{i} \in \mathcal{B}}} \rho_{\mathbf{k}_{1} \ldots \rho_{\mathbf{k}_{2 n}}} \delta_{\mathbf{k}_{1}+\ldots+\mathbf{k}_{2 n}}\right)(\mathrm{d} \rho)^{N}
\end{aligned}
$$

where $d(k)=a_{2}-\beta \Phi(k), \Phi(k)$ is the Fourier transform of the short-range interaction potential, $\mathcal{B}$ is Brillouin zone boundary and coefficients $a_{n}$ are some constants.

The partition function or the free energy has two different types of contributions: the energy contribution and the contribution which is connected with the interaction potential (the entropy contribution). The first one $\frac{1}{2} \sum_{\mathbf{k} \leqslant \mathcal{B}} \beta \Phi(k) \rho_{\mathbf{k}} \rho_{-\mathbf{k}}$ is diagonal in the $\rho_{\mathbf{k}}$-representation, and the entropy contribution $\sum_{\mathbf{l}}\left(\sum_{n \geqslant 0} \frac{a_{2 n}}{(2 n) !}\left(\rho_{\mathbf{l}}\right)^{2 n}\right)$ is diagonal in the $\rho_{\mathbf{l}}$-representation. So we can not calculate the partition function exactly. There are two ways, two possible approaches to calculate equation (5). The first one uses $\rho_{\mathbf{k}}$ variables and is connected with the application of the Gaussian moments, but in this case we have some condition $a_{2 n} \ll\left(a_{2}-\beta \Phi(k)\right)^{n}, n=2,3$. which is right far from the phase transition point and is not right near the phase transitions point, because the difference $a_{2}-\beta \Phi(k)$ turns to zero when $k \rightarrow 0$ and $T \rightarrow T_{\mathrm{c}}$.

The second way is connected with the $\rho_{\mathrm{l}}$-representation. In this case the entropy contribution is diagonal and for the energy contributions we use some approximations (Yukhnovskii approach: see I.R. Yukhnovskii. Phase Transitions of the Second Order: Collective Variables Method. World Scientific, Singapore, 1980). After some calculations we have obtained the following representation for the partition function:

$$
\begin{aligned}
Z & =C Z_{1} \ldots Z_{n-1} \int_{R_{n}}(\mathrm{~d} \rho)^{N_{n}} \exp \left[E_{n}(\rho)\right] \\
E_{n} & =-\frac{1}{2} \sum_{\mathbf{k} \in \mathcal{B}_{n}} d_{n}(k) \rho_{\mathbf{k}} \rho_{-\mathbf{k}}-\frac{1}{4 !} \frac{1}{N_{n}} \sum_{\substack{\mathbf{k}_{\mathbf{k}_{i}} \ldots \mathbf{k}_{4} \\
\mathbf{k}_{i} \in \mathcal{B}_{n}}} a_{n} \rho_{\mathbf{k}_{1} \ldots \rho_{\mathbf{k}_{4}}} \delta_{\mathbf{k}_{1}+\ldots+\mathbf{k}_{4}} .
\end{aligned}
$$


The quantity $Z_{n}$ is the partial partition function for the $N_{n}$ block structure and $E_{n}(\rho)$ is the microscopic analogue of Ginzburg-Landau Hamiltonian. But in the collective variables method we know the analytic expressions for the coefficients $d_{n}$ and $a_{n}$. If we want to calculate, for example, the free energy near the phase transition point we must take into account two different types of contributions. One part is connected with the renormalization group symmetry region and the other part corresponds to the limit Gaussian region (see figure 2). Some results of the calculation of the

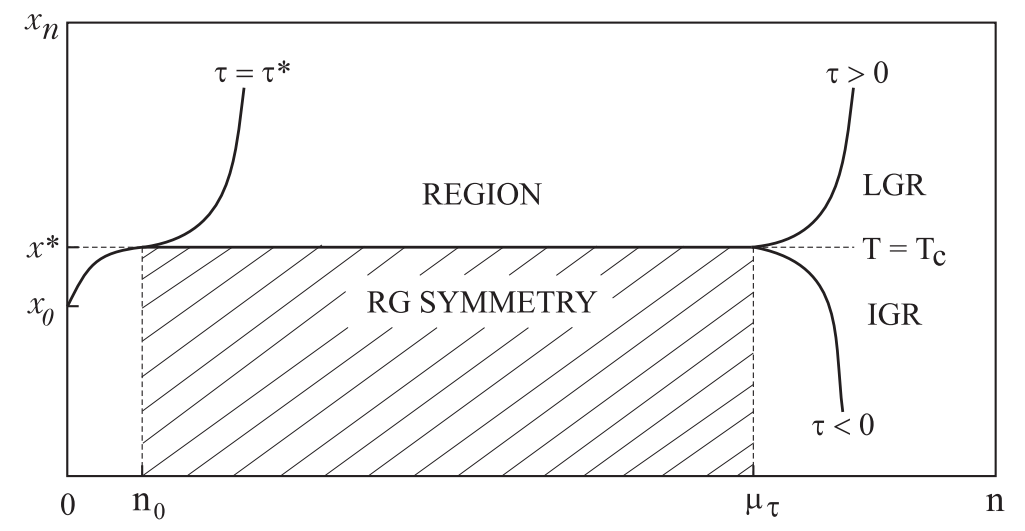

Figure 2. Evolution of the quantity $x_{n}=\sqrt{3}<d_{n}(k)>_{B_{n+1}, B_{n}} / \sqrt{a_{n}}$ with $n$ for temperatures close to $T_{\mathrm{c}}$.

specific heat of 3D one component model are shown in figure 3. You can see that the value (curve 2) which corresponds to the renormalization group contribution to the specific heat is negative. The critical exponent is correct. We have no problems with the calculation of the critical exponent, but the critical amplitude is negative. The contribution which corresponds to the limit Gaussian region (LGR) is shown by the curve 1. The specific heat critical exponent for this contribution is the same, but the sign of the critical amplitude is positive. So, we obtain the resulting curve (3) for the specific heat which has right critical exponent and right critical amplitude.

If you want to calculate only universal quantities we can use only the region where the renormalization group symmetry takes place. In the case one wants to calculate some non-universal properties, for instance, critical temperature, the size of the critical region, the critical amplitude and so on, one has to take into account the contribution from the limit Gaussian region in the case $T>T_{\mathrm{c}}$ and inverse Gaussian region (IGR) for $T<T_{\mathrm{c}}$.

Ihor Yukhnovskii. I would like to attract your attention. When we calculate the partition function by means of the quartic distribution function, it has the second degree and the forth degree of the collective variables of the density. We have two regions: the Gaussian region and the renormalization group one (see figure 2). In this renormalization group region we have to use only the quartic distribution function. But we cannot obtain right results when we work only in this region. The second small region at the end (see figure 2) is a limit Gaussian region. When the temperature turns to the critical one, this region shrinks to zero. But this region always gives a very big contribution to the free energy. And the specific heat becomes 


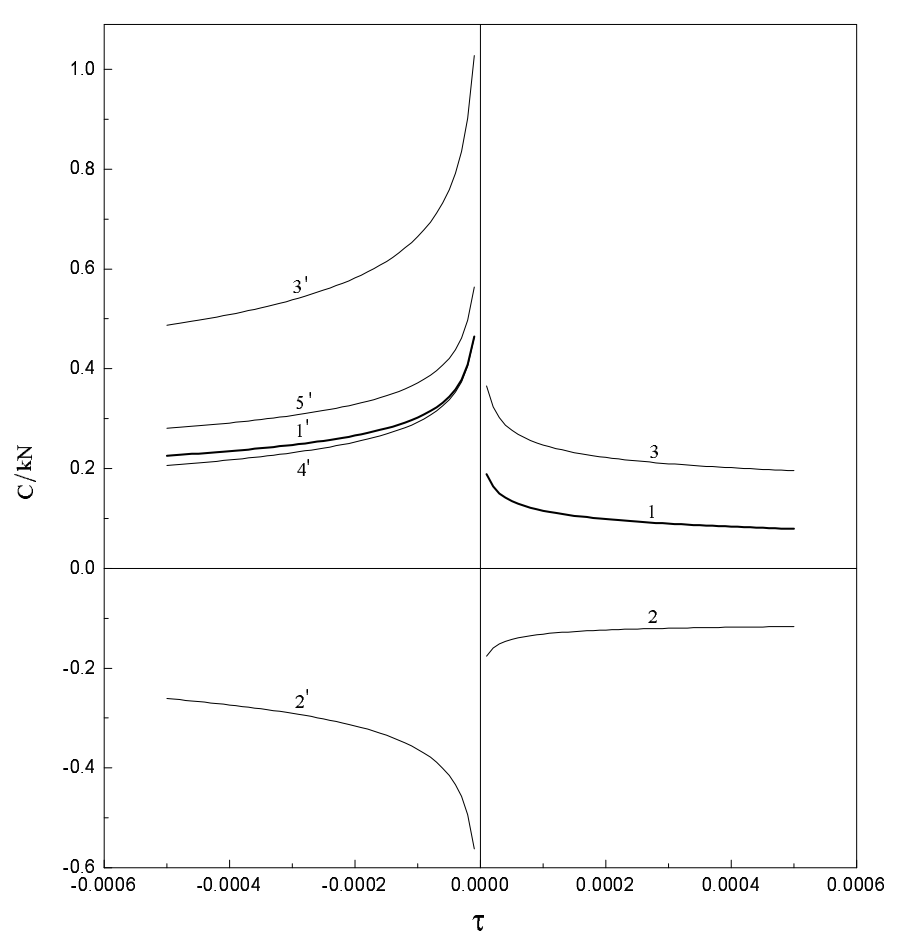

Figure 3. Heat capacity for small $\tau=\left(T-T_{\mathrm{c}}\right) / T_{\mathrm{c}}$.

positive. This is a very interesting point which I wanted to show you. So, I would like to repeat that first we must introduce a very rigorous mathematical apparatus. It can be very complicated, of course, but we have got a computer. Also we have a possibility to obtain the explicit expressions for thermodynamic functions of the models studied and to investigate their dependence on microscopic parameters, for example on the interaction parameters.

Michael Schick. What are you interested in? Are you interested in the criticality per se? Or you are interested in non-universal quantities?

Ihor Yukhnovskii. We want to obtain all: an equation of state, a free energy, an entropy, a susceptibility, critical indices. And all this is obtained from the first principles (you have to known only the interaction potential), in the framework of a unique approach.

Also, I want to tell you what we cannot obtain. We cannot obtain the solution for the $d=2$ model. Because when $d=2$ I have to take into account all terms in the Gibbs distribution. This means that this problem has an exact solution.

\section{There are possibilities not only to develop the theory but also to apply it...}

Alina Ciach. I don't want to continue this discussion, but I would like to draw your attention to very important, I think, and interesting problems which can be solved by applications of the theory of phase transitions to very complex systems such as 
biological systems.

The theory of phase transitions is developed quite well, it's improving and still developing. There are, however, interesting and important problems concerning complex systems, for example biological systems, which could be solved by applying the theory of phase transitions in its present form. The structure in biological cell or organella presumably depends on which phase should be stable in such complex systems in given conditions, and the structure determines the function or the phenomena which occur in such systems. There are even simple questions concerning phase transitions in complex systems, which are not yet solved. I don't want to describe the methods which should be used to describe the phase transitions in real biological systems. However, the structures formed in biological systems are very similar to the structures which spontaneously occur in self-assembling systems, such as lipids in water or surfactants in water or surfactants in water and oil mixtures, or co-polymers. In such systems solute particles are amphiphilic, and therefore selfassemble into monolayers or bilayers, to prevent unfavourable contacts between polar and nonpolar particles or particle parts. These monolayers or bilayers can arrange in very complex structures in different conditions. The size of the domains which are surrounded by these bilayers or monolayers is one or two orders of magnitude larger than molecular sizes. So, there is another length scale in the problem. An example

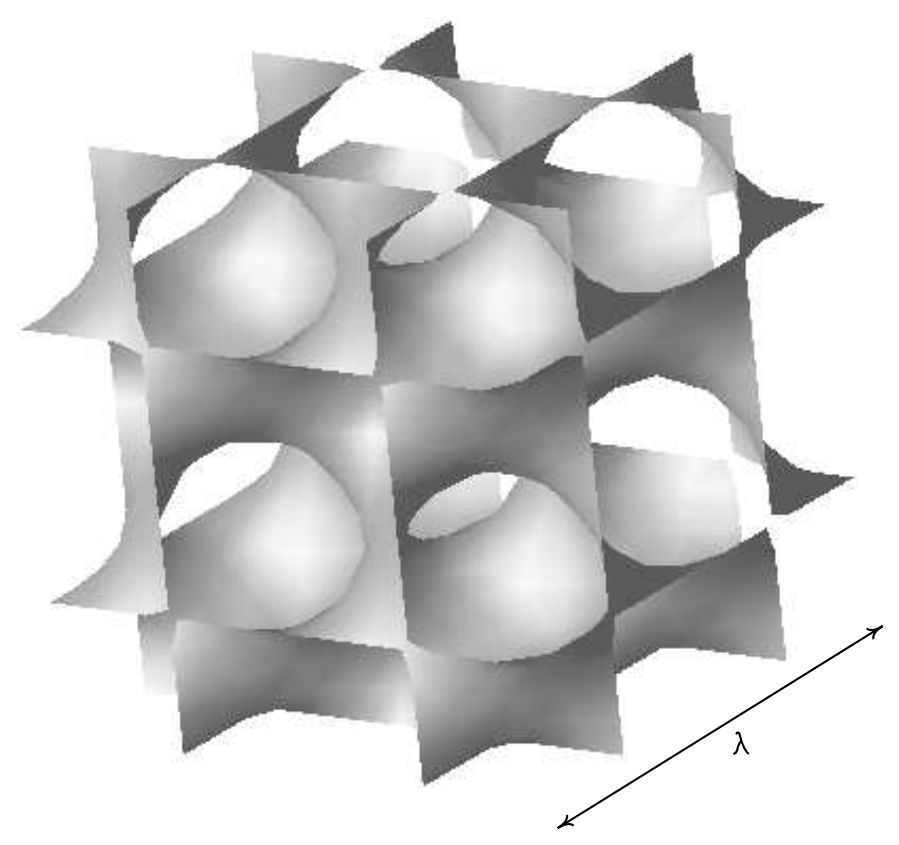

Figure 4. A unit cell of a cubic diamond phase.

of such a complex structure is the cubic diamond phase, which is periodic in space and whose unit cell is shown in figure 4. There are also different periodic phases, for example the giroid phase shown by Prof. Schick during his lecture. Endoplasmic reticulum, consisting of a phospholipid bilayer and extending over a large part of the cell cytoplasm, has such a complex structure. 
The phase behaviour in such systems is studied intensively in the recent years, however, the effect of surfaces or of confinement on such systems has not been studied so far very extensively. Confinement and boundaries certainly play an important role in biological systems, in particular for organella, which are not much larger than the distance between the bilayers in the endoplasmic reticulum.

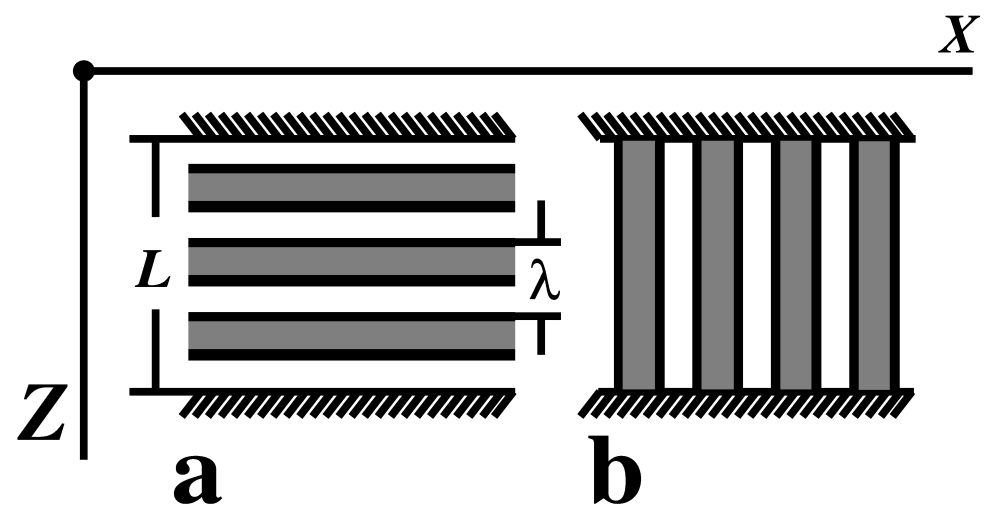

Figure 5. A lamellar phase confined in a slit with hydrophilic and neutral walls.

Now, what are the effects of various kinds of external surfaces or of confinement on such self-assembling systems? If we consider a slit geometry, for example, one can increase the slit size by a small fraction of the size of the unit cell. In figure 5 a lamellar phase confined in a slit with hydrophilic and neutral walls is shown. When the width $L$ of the slit is not commensurate with the size of the unit cell $\lambda$, the structure of the confined ordered phase will be deformed. And the question is, how this system responds to the stress which is present when $\lambda$ and $L$ do not match? What kinds of deformations will occur?

Condensation of a liquid-like phase in pores with adsorbing walls before the bulk phase transition takes place is a well known phenomenon in simple fluids. Are there similar capillary condensation phenomena for ordered periodic phases? How do they differ from the well known phenomena in uniform systems? The domains rich in one component and surrounded by amphiphiles play on the nanometer length scale a similar role as particles on a molecular length scale. Therefore some similarities between the behaviour of the simple fluids on the molecular length scale and selfassembling systems on the nanometer-length scale can be expected. On the other hand, there should also be differences, because the domains are not rigid but rather soft and compressible. Also, symmetry and anisotropy of such ordered phases may affect the phase transitions in confinement. The size and shape of pores can also have a strong effect on stability of various phases. It should be mentioned that unlike in simple fluids, there are many metastable phases of various structures on the nanometer length scale in such systems.

Wetting phenomena in such complex systems also deserve attention. In particular, you may ask whether the wetting phenomena on the nanometer length scale are the same as in simple fluids on the molecular length scale, or are they different? In particular, when you approach the phase coexistence between the lamellar and cubic 
phases, or between the lamellar and microemulsion phases, what will be the effect of the chemistry of the wall or of the symmetry and anisotropy of such phases? There are many questions and I think they offer large possibilities of applications of the theory of phase transitions. Even the simplest version of the theory, the mean field approximation, may give some insight into the problem. Just by applying the theory of the phase transitions to such complicated systems, you can describe different phenomena which have not been described. This is one example of the possibility of applications of the theory of phase transitions to fascinating problems, which are close to biological problems. So I liked to comment that there is not only a possibility to develop the theory, but also there is a vast possibility of its future applications.

Michael Schick. Also a theory of polymerizing liquids has huge biological implications. This is part of the theory of phase transitions. And we have not touched on it in any details.

Ihor Yukhnovskii. Biological systems are limited systems, and there should hold the mean field theory.

Michael Schick. That is certainly true for polymers (except in the vicinity of a critical point).

George Stell. Critical-point theory can be done by using the original Wilson approach or by using any one of several other approaches, as can the crossover problems mentioned by Doctors Folk, Chalyi, and Holovatch. Among the ones that can be incorporated into theories that remain useful far from the critical point, there is a systematic theory by Reatto and Parola and their co-workers. There is also work by John White, that follows more closely the original Wilson approach, and there is the development of Anisimov and Sengers and co-workers, which is more phenomenological but can be systematized and rigorized. Then there is the SCOZA theory that I am doing. And there are still others. All of these are different ways of approaching the same problem that the Lviv group is interested in. It is easy for each group to get so immersed in its own formalism that it loses sight of what the other groups are doing, and to begin to believe that it has THE right way to do things, rather than A right way. So I think each group has to try to pay attention to what the others are doing. Because each of these approaches has a somewhat different flavour than the others, with its own set of advantages and disadvantages. So I recommend that from time to time each group look around and see what the other people are doing.

Alexander Bakai. Biological systems in operating state are very sensitive to small changes in temperature, pressure, osmotic pressure etc. On the other hand they have to operate stably. And they are open systems which possess a set of stable dynamic and/or thermodynamic states. A dynamic system can change its attractor under action of a small perturbation only if it is close to an unstable point (e.g. saddle point). Thermodynamic system changes its phase state easy in the vicinity of critical point. In both cases fluctuations of order parameters are considerably large along with big response functions. How biological systems can combine a big sensitivity to perturbation (big response functions) with a high stability (small fluctuations of the order parameters)?

Wolffram Schröer. Biological systems work in non-equilibrium. 
Alina Ciach. I would like to comment on this effect of large response of a system to a small change of a control parameter. There are examples of such a behaviour which are not related to phase transitions, but rather to the structure of these complex systems.

Consider a lamellar phase like this (figure 5a) in a slit whose walls are strongly hydrophilic. Its orientation is parallel to the walls. On the other hand, when the wall is neutral, the surface tension between the wall and the lamellar phase in different orientations is almost the same. However, when the wall is weakly hydrophilic, the parallel orientation will be preferred for $\lambda$ and $L$ commensurate, and the perpendicular orientation will stabilize for slightly different $L$, when the width of the slit does not fit the period of the lamellar phase. In both cases the lamellar phase is stable, but the properties of the confined system are significantly different.

Yurij Holovatch. Thank you. Following the discussion I also tried to notice the fields which are traditionally related to phase transitions and critical phenomena but which were not mentioned here. Let me name some of them: we have not touched upon phase transitions on the surface which are intensively studied now both experimentally and theoretically (groups of Kurt Binder, Hans Werner Diehl and others). Polymers were mentioned here. Professor Schick in particular stated that mean field theory is good in explaining their properties, but this is when one studies the thermal behaviour and not too close to the critical point. Speaking about structural properties, in particular about scaling laws governing these properties one should rely on more refined theories such as the renormalization group approach which is traditionally used there since the pioneering works of Pierre Gilles de Gennes (let me mention here schools of Karl Freed, Lothar Schäfer). Professor Stell mentioned a very interesting class of objects: star polymers. These as well as polymer networks, copolymers and copolymer stars are the field of intensive studies now. One more generalization of polymer chains are membranes which themselves form an actively evolving part of physics. We were not speaking about quantum phase transitions which occur at zero temperature. A ruling parameter there is a change in a coupling. Professor Ciach as well as Professors Chalyi and Bakai mentioned a large response of a system to a small change of control parameter. This effect was exactly a reason to introduce one more class of models showing critical behaviour: the so-called SOC (self-organized criticality) models. These include forest fire models, traffic jam models etc. As I have told already the list is far from being complete. Returning to the start of our discussion: is the phase transition theory already completed or is it still developing? I think I will express common opinion if we allow both points of view to exist in replying to this question.

\section{Participants of the discussion}

Alexander Bakai, Prof. D.Sc., Kharkiv Institute of Physics \& Technology, Kharkiv, Ukraine

Alina Ciach, Prof. Dr. hab., Institute of Physical Chemistry, Polish Acad. Sci., Warszawa, Poland 
Alexander Chalyi, Prof. D.Sc., Department of Physics, O. Bohomolets' National Medical University, Kyiv, Ukraine

Reinhard Folk, a.o.Univ.Prof. Dr. hab., Johannes Kepler Universität Linz, Linz, Austria

Yurij Holovatch, D.Sc., Institute for Condensed Matter Physics, National Acad. Sci. of Ukraine, Lviv, Ukraine

Mykhajlo Kozlovskii, D.Sc., Institute for Condensed Matter Physics, National Acad. Sci. of Ukraine, Lviv, Ukraine

Michael Schick, Prof. Ph.D., Department of Physics, University of Washington Seattle, Washington, USA

Wolffram Schröer, Prof. Dr. hab., Institut für anorganische und physikalische Chemie, Universität Bremen, Bremen, Germany

George Stell, Prof. Ph.D, Department of Chemistry, State University of New York at Stony Brook, Stony Brook, USA

Ihor Yukhnovskii, Prof. D.Sc., Institute for Condensed Matter Physics, National Acad. Sci. of Ukraine, Lviv, Ukraine

Materials prepared by: Maxym Dudka, Yurij Holovatch, Roman Melnyk, Ihor Mryglod, Oksana Patsahan (ICMP), and Taras Yavors'kii (Ivan Franko National University of Lviv).

\section{Матеріали круглого столу “Фазові переходи і критичні явища: минуле, сучасне і майбутнє"}

У статті подано матеріали дискусії про фазові переходи та критичні явища, що відбулася підчас Наради із сучасних проблем теорії м'якої речовини (27-31 серпня 2000 р., м. Львів).

Ключові слова: фазові переходи, критичні явища

PACS: 01.30.Rr, 05.70.Fh, 64. 
